\title{
INDEX OF NATURAL OBJECTS
}

Achillea alpina, No. 3, 534

Angelica tenuissima Nakai (= Ligusticum tenuissimum

Kitagawa), No. 3, 529

Annona crassiflora, No. 3, 543

Aplysia pulmonica, No. 3, 557

Apocynum lancifolium, No. 3, 541

Artemisia abrotanum L., No. 3, 478

Artemisia argyi, No. 3, 414

Artemisia umbrosa Turcz. ex DC., No. 3, 545

Aspergillus sp. from Eucommia ulmoides, No. 3, 526

Betula birch, No. 3, 585

Cajanus cajan (L.) Millsp., No. 3, 438

Camelina sylvestris L., No. 3, 539

Caragana conferta Benth. ex Bake, No. 3, 440

Carthamus tinctorius L., No. 3, 427, 446

Climacoptera obtusifolia, No. 3, 537

Curcuma phaeocaulis Valeton, No. 3, 552

Dionysia oreodoxa Bornm., No. 3, 547

Dolomiaea souliei (Franch.) C. Shih, No. 3, 455

Echinothrix diadema, No. 3, 417
Ferula sinkiangensis, No. 3, 515

Galanthus gracilis Celak., No. 3, 573

Haematococcus pluvialis, No. 3, 578

Inonotus obliquus, No. 3, 524

Iris dichotoma Pall., No. 3, 430

Mortierella alpina, No. 3, 518

Panax ginseng C. A. Meyer, No. 3, 562, 565

Penicillium chrysogenum, No. 3, 405

Penicillium sp., No. 3, 568

Phomopsis sp. (No. Gx-4), No. 3, 424

Polygonatum glaberrimum K. Koch, No. 3, 489

Psammaplysilla purpurea, No. 3, 520

Rehmannia chingii, No. 3, 560

Scutellaria galericulata L., No. 3, 554

Setaria viridis, No. 3, 433

Silene colpophylla Wrigley, No. 3, 571

Streptomyces violans (strain HTTA-F04129), No. 3, 402

Suaeda salsa (L.) Pall., No. 3, 531

Tribulus terrestris L., No. 3, 483

Xylocarpus granatum K.D. Koenig, No. 3, 549 